\title{
Optimal Families of Eighth-Order Convergent Iterative Methods for Solving Nonlinear Equations Based on Ostrowsk's Method
}

\author{
N.M. Alrasheedi, I.A. Alsubiahi* \\ Department of mathematics, Science College, Taibah University, Saudi Arabia
}

Copyright $\bigcirc 2018$ by authors, all rights reserved. Authors agree that this article remains permanently open access under the terms of the Creative Commons Attribution License 4.0 International License

\begin{abstract}
Solving nonlinear equations is one of the most important problems in numerical analysis, and has a wide range of application in various aspects, as well as many branches of science, engineering, physics, computing, astronomy, finance, ... . Generally, it is difficult to find the exact root of the nonlinear equations, and so iterative methods become the efficient way to obtain approximate solutions. Recently Kou et. al. presented a class of new variants of Ostrowski's method with order of convergence equals seven (OSM7) for solving simple roots of nonlinear equations proposed in [7]. Ostrowski's method (OSM7) has efficiency index equals to $\sqrt[4]{7} \approx 1.626$, it has four functions per iteration but its order of convergence is seven that means it's not optimal method. In this paper, we have proposed new two improvements of Ostrowski's method (OSM7) to make it an optimal eight family and to increase its efficiency index. The first improvement has obtained by multiplied the third step of (OSM7) by product of two weight functions with some special conditions and the second improvement has obtained by multiplied the third step of (OSM7) by summation of two others weigh functions with special conditions, too. Using weight functions in every improvement helped us to improve the order of convergence of (OSM7) from seven to eight without changing the number of function evaluations to be an optimal family. New two optimal families have efficiency index equals to $\sqrt[4]{8} \approx 1.682$. Some numerical examples are provided to show the good performance of the new methods.
\end{abstract}

Keywords Convergence Order, Efficiency Index, Iterative Method, Nonlinear Equation

\section{Introduction}

Solving nonlinear equations is one of the most important problems in science and engineering but the exact and analytic solutions of such non-linear equations are not always easy to find. Due to their importance, many numerical iterative methods have been suggested and analyzed under certain conditions. Two important aspects related to iterative methods are order of convergence and computational efficiency. The efficiency index of an iterative method is defined as $p^{1 / m}$, where $p$ is the order of convergence and $m$ is the number of function evaluations per iteration [5].

In this paper, we develop an iterative method to find a simple root $\alpha$ of the nonlinear equation $f(\alpha)=0$, where $f: I \subseteq R \rightarrow R$ is a scalar function on an open interval $I$ and we consider that $f^{\prime}(\alpha) \neq 0$. It is well known that Newton's method (NM) is one of the best iterative methods and important method [5] to find the solution $\alpha$ by

$$
x_{n+1}=x_{n}-\frac{f\left(x_{n}\right)}{f^{\prime}\left(x_{n}\right)},
$$

which is quadratically convergent in the open interval $I$ that contains the exact root $\alpha$. Liu et. al. $[1,3,4,9,13]$ have worked on Ostrowski's method [15] and they improved it. Ostrowski's method with seventh-order convergence (OSM7) has considered by [7], given by:

$$
\begin{gathered}
\mathrm{y}_{\mathrm{n}}=\mathrm{x}_{\mathrm{n}}-\frac{\mathrm{f}\left(\mathrm{x}_{\mathrm{n}}\right)}{\mathrm{f}^{\prime}\left(\mathrm{x}_{\mathrm{n}}\right)^{\prime}} \\
z_{n}=y_{n}-\frac{f\left(x_{n}\right)}{f\left(x_{n}\right)-2 f\left(y_{n}\right)} \frac{f\left(y_{n}\right)}{f^{\prime}\left(x_{n}\right)}, \\
x_{n+1}=z_{n}-\frac{f\left(z_{n}\right)}{f^{\prime}\left(x_{n}\right)}\left[\frac{\left(f\left(x_{n}\right)-f\left(y_{n}\right)\right)^{2}}{\left(f\left(x_{n}\right)-2 f\left(y_{n}\right)\right)^{2}}+\frac{f\left(z_{n}\right)}{f\left(y_{n}\right)-\theta f\left(z_{n}\right)}\right],
\end{gathered}
$$

where $\theta$ is constant.

According to the optimality, the optimal order of any multipoint iterative method is given by $2^{m-1}[8,10]$, where $m$ is the number of functional evaluations per iteration. It is clear that this variant requires to be of order eight to be an optimal iterative method, so we multiply the last step by two weight functions which they satisfy some conditions and without using more evaluations. These weight functions increased the order of this method from seven to eight in order to be an optimal iterative method, see [2]. 


\section{Construction of the New Optimal Family of Iterative methods}

Based on the family of iterative method (1), we constructed new two optimal families of iterative methods by using two weight functions as follows:

The first improvement of scheme (1), $\left(N S M_{1}\right)$ :

$$
\begin{gathered}
y_{n}=x_{n}-\frac{f\left(x_{n}\right)}{f^{\prime}\left(x_{n}\right)}, \\
z_{n}=y_{n}-\frac{f\left(x_{n}\right)}{f\left(x_{n}\right)-2 f\left(y_{n}\right)} \frac{f\left(y_{n}\right)}{f^{\prime}\left(x_{n}\right)} \\
x_{n+1}=z_{n}-\frac{f\left(z_{n}\right)}{f^{\prime}\left(x_{n}\right)} \times\{A(v) B(s)\} \\
\times\left[\frac{\left(f\left(x_{n}\right)-f\left(y_{n}\right)\right)^{2}}{\left(f\left(x_{n}\right)-2 f\left(y_{n}\right)\right)^{2}}+\frac{f\left(z_{n}\right)}{f\left(y_{n}\right)-\theta f\left(z_{n}\right)}\right]
\end{gathered}
$$

where $A(v)$ and $B(s)$ are two real weight functions with $v=\frac{f\left(z_{n}\right)}{f\left(x_{n}\right)}, s=\frac{f\left(z_{n}\right)}{f\left(y_{n}\right)}$ and $\theta \in \mathrm{R}$ is a constant.

The order of convergence of the preceding method is analyzed in theorem 1 .

\section{Theorem 1}

Let $\alpha \in I$ be a simple zero of a sufficiently differentiable function $f: I \rightarrow R$ for an open interval I. If $x_{0}$ is sufficiently close to $\alpha$, then the method (2) gives optimal of eight-order of convergence when $A(0)=1, A^{\prime}(0)=4, B(0)=1$, $B^{\prime}(0)=0$, and $\left|B^{\prime \prime}(0)\right|<\infty$.

Proof. Let $\alpha$ be a simple zero of equation and $x_{n}=\alpha+$ $e$. By using Taylor expansion, we have

$$
\begin{gathered}
f(x)=f^{\prime}(\alpha)\left(\begin{array}{c}
e+c_{2} \mathrm{e}^{2}+c_{3} \mathrm{e}^{3}+\mathrm{c}_{4} \mathrm{e}^{4}+ \\
+c_{5} \mathrm{e}^{5}+\mathrm{c}_{6} \mathrm{e}^{6}+\mathrm{c}_{7} \mathrm{e}^{7}+\mathrm{c}_{8} \mathrm{e}^{8}+\mathrm{c}_{9} \mathrm{e}^{9}
\end{array}\right) . \\
f^{\prime}(x)= \\
=f^{\prime}(\alpha)\left(\begin{array}{c}
1+2 c_{2} e+3 c_{3} e^{2}+4 c_{4} e^{3}+5 c_{5} e^{4}+ \\
+6 c_{6} e^{5}+7 c_{7} e^{6}+8 c_{8} e^{7}+9 c_{9} e^{8}
\end{array}\right)
\end{gathered}
$$

where $c_{k}=\frac{1}{k !} \frac{f^{(k)}}{f \prime(\alpha)} \quad$ for $\mathrm{k}=2,3, \ldots$.

Dividing (3) by (4) to get

$\frac{f(x)}{f^{\prime}(x)}=e-c_{2} e^{2}+\left(2 c_{2}^{2}-2 c_{3}\right) e^{3}+\ldots+\left(-64 c_{2}^{7}+\right.$ $\left.304 c_{2}^{5} c_{3}-176 c_{2}^{4} c 4-\cdots+31 c_{4} c_{5}-7 c_{8}\right) e^{8}+$ $O\left(e^{9}\right)$.

By expanding $y_{n}$ about $\alpha$, we obtain

$$
y_{n}=\left(\alpha+c_{2} e^{2}+\left(-2 c_{2}^{2}+2 c_{3}\right) e^{3}+\cdots+O\left(e^{9}\right) .\right.
$$

Expanding $f\left(y_{n}\right)$ about $\alpha$ to get

$$
\begin{gathered}
f\left(y_{n}\right)=f^{\prime}(\alpha) \mathrm{c}_{2} \mathrm{e}^{2}+\left(-2 f^{\prime}(\alpha) \mathrm{c}_{2}^{2}+2 f^{\prime}(\alpha) \mathrm{c}_{3}\right) \mathrm{e}^{3}+ \\
+\left(\begin{array}{c}
5 f^{\prime}(\alpha) \mathrm{c}_{2}^{3}-7 f^{\prime}(\alpha) \mathrm{c}_{2} \mathrm{c}_{3}+ \\
+3 f^{\prime}(\alpha) \mathrm{c}_{4}
\end{array}\right) \mathrm{e}^{4}+\cdots+O\left(e^{9}\right) .
\end{gathered}
$$

By substituting (3), (4), (5) and (6) into the second formula of (2), using Taylor's expansion, and expanding, we obtain

$$
\begin{gathered}
z_{n}=\alpha+\left(4 c_{2}^{6}-8 c_{2}^{4} c_{3}+4 c_{2}^{2} c_{3}^{2}\right) \mathrm{e}^{7}+ \\
+\left(\begin{array}{c}
-c_{2}^{7} \theta-31 c_{2}^{7}+3 c_{2}^{5} c_{3} \theta+92 c_{2}^{5} c_{3}-3 c_{2}^{3} c_{3}^{2} \theta- \\
-15 c_{2}^{4} c_{4}-76 c_{2}^{3} c_{3}^{2}+c_{2} c_{3}^{3} \theta+15 c_{2}^{2} c_{3} c_{4}+15 c_{2} c_{3}^{3}
\end{array}\right) e^{8} \\
+\cdots+O\left(e^{9}\right) .
\end{gathered}
$$

Expanding $f\left(z_{n}\right)$ about $\alpha$ to get

$$
f\left(z_{n}\right)=\left(f^{\prime}(\alpha) \mathrm{c}_{2}^{3}-f^{\prime}(\alpha) \mathrm{c}_{2} c_{3}\right) \mathrm{e}^{4}+\ldots+O\left(e^{9}\right) .
$$

Expanding $A(v)$ and $B(s)$ about 0 yields

$$
\begin{aligned}
& A(v)=A(0)+A^{\prime}(0) v+A^{\prime \prime}(0) \frac{v}{2 !}+ \\
& +A^{\prime \prime \prime}(0) \frac{v^{3}}{3 !}+A^{\prime \prime \prime \prime}(0) \frac{v^{4}}{4 !}+\cdots+O\left(e^{9}\right) . \\
& B(s)=B(0)+B^{\prime}(0) s+B^{\prime \prime}(0) \frac{s}{2 !}+ \\
& +B^{\prime \prime \prime}(0) \frac{s^{3}}{3 !}+B^{\prime \prime \prime \prime}(0) \frac{s^{4}}{4 !}+\ldots+O\left(e^{9}\right) .
\end{aligned}
$$

Substituting (3), (4), (6) - (8) and the two weight functions $A(v)$ and $B(s)$ into the third step of the formula to get $x_{n+1}=\alpha+\left(-c_{2}^{7} \theta+5 c_{2}^{7}+3 c_{2}^{5} c_{3} \theta-12 c_{2}^{5} c_{3}-\right.$ $3 c_{2}^{3} c_{3}^{2} \theta+c_{2}^{4} c_{4}+8 c_{2}^{3} c_{3}^{2}+c_{2} c_{3}^{3} \theta-c_{2}^{2} c_{3} c_{4}-$ $\left.c_{2} c_{3}^{3}\right) e^{8}+O\left(e^{9}\right)$, (9)

which indicates that the order of convergence of the family in (2) is eighth for any value of $\theta$. This completes the proof.

As we see from theorem 1 that the choice of the weight function is so important to make the order of the proposed class of iterative methods, see [3,11].

Now, we suggest the following new iterative methods by using the weight functions that satisfy the previous conditions in theorem 1.

Method (NS1): Consider

$A(v)=e^{4 v}$,

$B(s)=\cos (s)$, then

$y_{n}=x_{n}-\frac{f\left(x_{n}\right)}{f^{\prime}\left(x_{n}\right)}$

$z_{n}=y_{n}-\frac{f\left(x_{n}\right)}{f\left(x_{n}\right)-2 f\left(y_{n}\right)} \frac{f\left(y_{n}\right)}{f^{\prime}\left(x_{n}\right)}$,

$x_{n+1}=z_{n}-\frac{f\left(z_{n}\right)}{f^{\prime}\left(x_{n}\right)} \times\left\{e^{4 v} \cos (s)\right\}$

$\times\left[\frac{\left(f\left(x_{n}\right)-f\left(y_{n}\right)\right)^{2}}{\left(f\left(x_{n}\right)-2 f\left(y_{n}\right)\right)^{2}}+\frac{f\left(z_{n}\right)}{f\left(y_{n}\right)-\theta f\left(z_{n}\right)}\right]$.

\section{Method (NS2): Consider}

$$
\begin{aligned}
A(v)= & \cos (v)+4 v+a_{1} v^{a}, \text { where } a \geq 2 \text { and } \\
& a_{1} \in R, \\
B(s)= & b_{1} s^{b}+1, \text { where } b \geq 3 \text { and } b_{1} \in R, \text { then }
\end{aligned}
$$

$y_{n}=x_{n}-\frac{f\left(x_{n}\right)}{f^{\prime}\left(x_{n}\right)}$

$z_{n}=y_{n}-\frac{f\left(x_{n}\right)}{f\left(x_{n}\right)-2 f\left(y_{n}\right)} \frac{f\left(y_{n}\right)}{f^{\prime}\left(x_{n}\right)}$,

$x_{n+1}=z_{n}-\frac{f\left(z_{n}\right)}{f^{\prime}\left(x_{n}\right)} \times\left\{\begin{array}{c}\left(\cos (v)+4 v+a_{1} v^{a}\right) \\ \left(b_{1} s^{b}+1\right)\end{array}\right\}$ 


$$
\times\left[\frac{\left(f\left(x_{n}\right)-f\left(y_{n}\right)\right)^{2}}{\left(f\left(x_{n}\right)-2 f\left(y_{n}\right)\right)^{2}}+\frac{f\left(z_{n}\right)}{f\left(y_{n}\right)-\theta f\left(z_{n}\right)}\right] .
$$

Second improvement of scheme (1), (NSM $)$ :

$$
\begin{gathered}
y_{n}=x_{n}-\frac{f\left(x_{n}\right)}{f^{\prime}\left(x_{n}\right)}, \\
x_{n+1}=y_{n}-\frac{f\left(x_{n}\right)}{f\left(x_{n}\right)-2 f\left(y_{n}\right)} \frac{f\left(y_{n}\right)}{f^{\prime}\left(x_{n}\right)}, \\
x_{n+1}=z_{n}-\frac{f\left(z_{n}\right)}{f^{\prime}\left(x_{n}\right)} \times\{A(v)+B(s)\} \times \\
\times\left[\frac{\left(f\left(x_{n}\right)-f\left(y_{n}\right)\right)^{2}}{\left(f\left(x_{n}\right)-2 f\left(y_{n}\right)\right)^{2}}+\frac{f\left(z_{n}\right)}{f\left(y_{n}\right)-\theta f\left(z_{n}\right)}\right],
\end{gathered}
$$

where $A(v)$ and $B(s)$ are two real weight functions with $v=\frac{f\left(z_{n}\right)}{f\left(x_{n}\right)}$ and $s=\frac{f\left(z_{n}\right)}{f\left(y_{n}\right)}$ and $\theta \in \mathrm{R}$ is constant.

The order of convergence of the preceding method is analyzed in the following theorem.

\section{Theorem 2}

Let $\alpha \in I$ be a simple zero of a sufficiently differentiable function $f: I \subseteq R \rightarrow R$ for an open interval I. If $x_{0}$ is sufficiently close to $\alpha$, then the method (10) gives optimal eight-order of convergence when $A(0)=1, A^{\prime}(0)$ $=4, B(0)=0, B^{\prime}(0)=0$, and $B^{\prime \prime}(0)=0$.

Proof. Substituting (3), (4), (6)-(8), and the two weight functions $A(v)$ and $B(s)$ into the third step of the (10) formula to get

$$
\begin{gathered}
x_{n+1}=\alpha+ \\
+\left(\begin{array}{c}
-c_{2}^{7} \theta+5 c_{2}^{7}+3 c_{2}^{5} c_{3} \theta-12 c_{2}^{5} c_{3}-3 c_{2}^{3} c_{3}^{2} \theta+ \\
+c_{2}^{4} c_{4}+8 c_{2}^{3} c_{3}^{2}+c_{2} c_{3}^{3} \theta-c_{2}^{2} c_{3} c_{4}-c_{2} c_{3}^{3}
\end{array}\right) e^{8} \\
+O\left(e^{9}\right)
\end{gathered}
$$

Same for method (2) that the order of convergence of the family in (10) is eighth for any value of $\theta$. This completes the proof.

Now, with special weight functions which they satisfy the conditions in theorem 2 we obtain

Method (NS3): Consider

$A(v)=1+4 v+c_{1} v^{c}$, where $c \geq 2$ and $c_{1} \in R$,

$B(s)=d_{1} s^{d}$, where $d \geq 3$ and $d_{1} \in R$, then

$y_{n}=x_{n}-\frac{f\left(x_{n}\right)}{f^{\prime}\left(x_{n}\right)}$

$z_{n}=y_{n}-\frac{f\left(x_{n}\right)}{f\left(x_{n}\right)-2 f\left(y_{n}\right)} \frac{f\left(y_{n}\right)}{f^{\prime}\left(x_{n}\right)^{\prime}}$

$x_{n+1}=z_{n}-\frac{f\left(z_{n}\right)}{f^{\prime}\left(x_{n}\right)} \times\left\{1+4 v+c_{1} v^{c}+d_{1} s^{d}\right\}$

$\times\left[\frac{\left(f\left(x_{n}\right)-f\left(y_{n}\right)\right)^{2}}{\left(f\left(x_{n}\right)-2 f\left(y_{n}\right)\right)^{2}}+\frac{f\left(z_{n}\right)}{f\left(y_{n}\right)-\theta f\left(z_{n}\right)}\right]$.

\section{Method (NS4): Consider}

$A(v)=\frac{2-v}{2-9 v}$

$B(s)=s^{m} e^{s}$, where $m \geq 3$ and $s \in R$, then

$$
\begin{aligned}
& y_{n}=x_{n}-\frac{f\left(x_{n}\right)}{f^{\prime}\left(x_{n}\right)^{\prime}}, \\
& z_{n}=y_{n}-\frac{f\left(x_{n}\right)}{f\left(x_{n}\right)-2 f\left(y_{n}\right)} \frac{f\left(y_{n}\right)}{f^{\prime}\left(x_{n}\right)^{\prime}} \\
& x_{n+1}=z_{n}-\frac{f\left(z_{n}\right)}{f^{\prime}\left(x_{n}\right)} \times\left\{\frac{2-v}{2-9 v}+s^{m} e^{s}\right\} \\
& \times\left[\frac{\left(f\left(x_{n}\right)-f\left(y_{n}\right)\right)^{2}}{\left(f\left(x_{n}\right)-2 f\left(y_{n}\right)\right)^{2}}+\frac{f\left(z_{n}\right)}{f\left(y_{n}\right)-\theta f\left(z_{n}\right)}\right] .
\end{aligned}
$$

\section{Numerical Results}

In this section, we compared the performance of the fourth cases of the new optimal eighth-order methods (2) and (10) with some famous iterative methods. In case NS1 and NS3 we take $\theta=0,0.5,1,-1$ but in case NS2 and NS4 we only take $\theta=0$ and we take $a_{1}=b_{1}=c_{1}=$ $d_{1}=0$ and for method NS4 we take $m=3$. We compared our methods with the original methods OSM7(1) and some known methods as follows:

Newton's method (NM):

$y_{n}=x_{n}-\frac{f\left(x_{n}\right)}{f^{\prime}\left(x_{n}\right)}$.

King's method (KM) [6]:

$y_{n}=x_{n}-\frac{f\left(x_{n}\right)}{f^{\prime}\left(x_{n}\right)}$

$x_{n+1}=z_{n}-\frac{f\left(x_{n}\right)+\theta f\left(y_{n}\right)}{f\left(x_{n}\right)+(\theta-2) f\left(y_{n}\right)} \frac{f\left(y_{n}\right)}{f^{\prime}\left(x_{n}\right)}$,

where $\theta=0$.

Ostrowski's method of order eighth (OSM8) [14]:

$y_{n}=x_{n}-\frac{f\left(x_{n}\right)}{f^{\prime}\left(x_{n}\right)}$

$z_{n}=y_{n}-\frac{f\left(x_{n}\right)}{f\left(x_{n}\right)-2 f\left(y_{n}\right)} \frac{f\left(y_{n}\right)}{f^{\prime}\left(x_{n}\right)^{\prime}}$

$x_{n+1}=z_{n}-\left(\sin \left(\frac{f\left(z_{n}\right)}{f\left(x_{n}\right)}\right)+\cos \left(\frac{f\left(z_{n}\right)}{f\left(x_{n}\right)}\right)\right)$

$\times \frac{f\left(z_{n}\right) f\left[x_{n}, y_{n}\right]}{f\left[x_{n}, z_{n}\right] f\left[y_{n}, z_{n}\right]} \times\left(1+\frac{f^{4}\left(y_{n}\right)}{f^{4}\left(x_{n}\right)} e^{\frac{f\left(y_{n}\right)}{f\left(x_{n}\right)}}\right) e^{\frac{f\left(z_{n}\right)^{2}}{f\left(y_{n}\right)^{2}}}$,

where $f\left[x_{n}, z_{n}\right]$ and $f\left[y_{n}, z_{n}\right]$ are divided differences.

Sharma-Arora's method of order eight (SAM8) [12]:

$y_{n}=x_{n}-\frac{f\left(x_{n}\right)}{f^{\prime}\left(x_{n}\right)}$,

$z_{n}=y_{n}-\frac{f\left(y_{n}\right)}{2 f\left[x_{n}, y_{n}\right]-f^{\prime}\left(x_{n}\right)}$

$x_{n+1}=z_{n}-\frac{f\left[y_{n}, z_{n}\right]}{f\left[x_{n}, z_{n}\right]} \frac{f\left(z_{n}\right)}{2 f\left[y_{n}, z_{n}\right]-f\left[x_{n}, z_{n}\right]}$.

where $f\left[x_{n}, y_{n}\right]$ is a divided difference.

In Table 1, the efficiency index of (2) and (10) equals to $\sqrt[4]{8} \approx 1.682$ it is higher than the efficiency index of OSM 7 which is $\sqrt[4]{7} \approx 1.626$. 
Table 1. Order of convergence, number of function evaluations and

\begin{tabular}{cccc}
\multicolumn{5}{c}{ Efficiency index. } \\
Method & $\mathbf{P}$ & $\mathbf{N}$ & EI \\
NM & 2 & 2 & $\sqrt[2]{2} \approx 1.1414$ \\
KM & 4 & 3 & $\sqrt[3]{4} \approx 1.587$ \\
OSM7 & 7 & 4 & $\sqrt[4]{7} \approx 1.626$ \\
OSM8 & 8 & 4 & $\sqrt[4]{8} \approx 1.682$ \\
$\mathrm{NSM}_{1}$ & 8 & 4 & $\sqrt[4]{8} \approx 1.682$ \\
$\mathrm{NSM}_{2}$ & 8 & 4 & $\sqrt[4]{8} \approx 1.682$ \\
$\mathrm{SAM}_{8}$ & 8 & 3 & $\sqrt[3]{8}=2$
\end{tabular}

We using the following examples to show the effectiveness of the new methods:

$$
f_{1}(x)=x^{2}-(1-x)^{25} \quad \alpha=0.1437392592995
$$

$$
\begin{array}{ll}
f_{2}(x)=x^{3}+\log (+x) & \alpha=3.7189222 e-1009 \\
f_{3}(x)=\sin (x)-\frac{x}{3} & \alpha=1178862600758 \\
f_{4}(x)=e^{x}+x-20 & \alpha=2.842438953784
\end{array}
$$

The MATLAB program is used for all the computations using 1000 digit. Table 2 presents the number of iterations to approximate the zero (IT) and contains the value of $\left|f\left(x_{n}\right)\right|,\left|x_{n+1}-x_{n}\right|$ and COC. The values of the computational order of convergence (COC) may be approximated ascoc $\approx \frac{\ln \left|\left(x_{n+1}-\alpha\right) /\left(x_{n}-\alpha\right)\right|}{\ln \left|\left(x_{n}-\alpha\right) /\left(x_{n-1}-\alpha\right)\right|}$. The following

\begin{tabular}{|c|c|c|c|c|c|c|c|c|c|}
\hline Method & IT & $\left|x_{n+1}-x_{n}\right|$ & $\left|f\left(x_{n}\right)\right|$ & $\mathrm{COC}$ & Method & IT & $\left|x_{n+1}-x_{n}\right|$ & $\left|f\left(x_{n}\right)\right|$ & $\mathrm{COC}$ \\
\hline \multicolumn{5}{|c|}{$f_{1}(x)=x^{2}-(1-x)^{25}$} & \multicolumn{5}{|c|}{$f_{2}(x)=x^{3}+\log (1+x)$} \\
\hline NM & 7 & $7.21 \mathrm{e}-22$ & $3.88 \mathrm{e}-42$ & 2 & $\mathrm{NM}$ & 5 & $1.39 \mathrm{e}-19$ & $9.68 \mathrm{e}-39$ & 2.00001 \\
\hline KM & 4 & $5.54 \mathrm{e}-21$ & $1.05 \mathrm{e}-79$ & 4 & $\mathrm{KM}$ & 4 & $9.64 \mathrm{e}-53$ & $4.76 \mathrm{e}-209$ & 4 \\
\hline $\mathrm{NS} 1, \theta=0$ & 4 & $7.75 \mathrm{e}-73$ & $7.62 e-572$ & 8 & $\mathrm{NS} 1, \theta=0$ & 3 & $1.36 \mathrm{e}-42$ & $2.89 \mathrm{e}-336$ & 8 \\
\hline $\mathrm{NS} 1, \theta=0.5$ & 4 & $4.19 \mathrm{e}-78$ & $5.73 e-614$ & 7.9606 & $\mathrm{NS} 1, \theta=0.5$ & 3 & $1.37 \mathrm{e}-44$ & $8.04 \mathrm{e}-353$ & 8.143 \\
\hline $\mathrm{NS} 1, \theta=1$ & 4 & $1.39 \mathrm{e}-81$ & $8.49 \mathrm{e}-642$ & 7.9685 & $\mathrm{NS} 1, \theta=1$ & 3 & $7.12 \mathrm{e}-46$ & $2.53 e-362$ & 8 \\
\hline $\mathrm{NS} 1, \theta=-1$ & 5 & $2.22 \mathrm{e}-68$ & $3.24 \mathrm{e}-536$ & 8 & $\mathrm{NS} 1, \theta=-1$ & 3 & $3.80 \mathrm{e}-40$ & $3.94 \mathrm{e}-316$ & 8 \\
\hline $\mathrm{NS} 2, \theta=0$ & 4 & $5.11 \mathrm{e}-69$ & $2.73 e-541$ & 8 & $\mathrm{NS} 2, \theta=0$ & 3 & $8.23 \mathrm{e}-46$ & $1.95 \mathrm{e}-362$ & 8 \\
\hline $\mathrm{NS} 3, \theta=0$ & 4 & $2.79 \mathrm{e}-73$ & $2.19 \mathrm{e}-575$ & 8 & $\mathrm{NS} 3, \theta=0$ & 3 & $1.28-44$ & $4.84 \mathrm{e}-353$ & 8.0001 \\
\hline $\mathrm{NS} 3, \theta=0.5$ & 4 & $5.47 \mathrm{e}-79$ & $5.75 e-621$ & 8 & $\mathrm{NS} 3, \theta=0.5$ & 3 & $3.51-46$ & $8.85 e-365$ & 8 \\
\hline $\mathrm{NS} 3, \theta=1$ & 4 & $8.28 \mathrm{e}-83$ & $1.38 \mathrm{e}-651$ & 8 & $\mathrm{NS} 3, \theta=1$ & 3 & $1.67-53$ & $4.24 \mathrm{e}-423$ & 8 \\
\hline $\mathrm{NS} 3, \theta=-1$ & 5 & $2.03 \mathrm{e}-66$ & $75 e-520$ & 8 & $\mathrm{NS} 3, \theta=-1$ & 3 & $4.57-41$ & $1.10 \mathrm{e}-323$ & 8.0107 \\
\hline $\mathrm{NS} 4, \theta=0$ & 4 & $1.36 \mathrm{e}-72$ & $7.22 \mathrm{e}-570$ & 8 & $\mathrm{NS} 4, \theta=0$ & 3 & $2.00 \mathrm{e}-44$ & $1.67 \mathrm{e}-351$ & 8.00001 \\
\hline OSM7 & 4 & $9.91 \mathrm{e}-89$ & $5.24 \mathrm{e}-612$ & 7 & OSM7 & 3 & $3.74 \mathrm{e}-39$ & $1.20 \mathrm{e}-269$ & 3.2467 \\
\hline OSM8 & 4 & $5.95 \mathrm{e}-49$ & $1.70 \mathrm{e}-380$ & 8 & OSM8 & 3 & $1.81 \mathrm{e}-43$ & $1.41 \mathrm{e}-342$ & 8 \\
\hline SAM8 & 4 & $1.84 \mathrm{e}-89$ & $3.94 \mathrm{e}-705$ & 8 & SAM8 & 3 & $4.85 \mathrm{e}-44$ & $2.74 \mathrm{e}-347$ & \\
\hline \multicolumn{5}{|c|}{$f_{3}(x)=\sin (x)-\frac{x}{3}$} & \multicolumn{5}{|c|}{$f_{4}(x)=e^{x}+x-20$} \\
\hline $\mathrm{NM}$ & 4 & 7.192e-16 & $1.96 \mathrm{e}-31$ & 1.9995 & NM & 6 & $4.49 \mathrm{e}-18$ & $1.74 \mathrm{e}-34$ & 2 \\
\hline KM & 4 & $9.241 \mathrm{e}-53$ & $7.18 \mathrm{e}-210$ & 4 & $\mathrm{KM}$ & 4 & $6.10 \mathrm{e}-45$ & $7.81 \mathrm{e}-178$ & 4 \\
\hline $\mathrm{NS} 1, \theta=0$ & 3 & $2.31 \mathrm{e}-45$ & $2.10 \mathrm{e}-359$ & 8 & $\mathrm{NS} 1, \theta=0$ & 3 & $1.42 \mathrm{e}-39$ & $4.59 \mathrm{e}-313$ & 8 \\
\hline $\mathrm{NS} 1, \theta=0.5$ & 3 & $7.61 \mathrm{e}-46$ & $6.47 \mathrm{e}-368$ & 8 & $\mathrm{NS} 1, \theta=0.5$ & 3 & $1.02 \mathrm{e}-39$ & $3.25 \mathrm{e}-314$ & 8 \\
\hline $\mathrm{NS} 1, \theta=1$ & 3 & $2.07-46$ & $6.47 e-368$ & 8 & $\mathrm{NS} 1, \theta=1$ & 3 & $7.27 e-40$ & $1.95 \mathrm{e}-315$ & 8 \\
\hline $\mathrm{NS} 1, \theta=-1$ & 3 & $1.44 \mathrm{e}-44$ & $5.43 e-353$ & 8 & $\mathrm{NS} 1, \theta=-1$ & 3 & $2.58 \mathrm{e}-39$ & $5.88 \mathrm{e}-311$ & 8 \\
\hline $\mathrm{NS} 2, \theta=0$ & 3 & $3.46 \mathrm{e}-45$ & $5.25 \mathrm{e}-358$ & 8 & $\mathrm{NS} 2, \theta=0$ & 3 & $1.98 \mathrm{e}-39$ & $6.50 \mathrm{e}-312$ & 8 \\
\hline $\mathrm{NS} 3, \theta=0$ & 3 & $1.47 \mathrm{e}-45$ & $4.74 \mathrm{e}-361$ & 8 & $\mathrm{NS} 3, \theta=0$ & 3 & $1.13 \mathrm{e}-39$ & $7.38 \mathrm{e}-314$ & 8 \\
\hline $\mathrm{NS} 3, \theta=0.5$ & 3 & $4.51 \mathrm{e}-46$ & $3.29-365$ & 8 & $\mathrm{NS} 3, \theta=0.5$ & 3 & $8.15 \mathrm{e}-40$ & $4.87 \mathrm{e}-315$ & 8 \\
\hline $\mathrm{NS} 3, \theta=1$ & 3 & $1.13 \mathrm{e}-46$ & $4.08 \mathrm{e}-370$ & 8 & $\mathrm{NS} 3, \theta=1$ & 3 & $5.71 \mathrm{e}-40$ & $2.70 \mathrm{e}-316$ & 8 \\
\hline $\mathrm{NS} 3, \theta=-1$ & 3 & $9.94 \mathrm{e}-45$ & $2.75 \mathrm{e}-354$ & 8 & $\mathrm{NS} 3, \theta=-1$ & 3 & $2.09 \mathrm{e}-39$ & $1.07 \mathrm{e}-311$ & 8 \\
\hline $\mathrm{NS} 4, \theta=0$ & 3 & $5.42 \mathrm{e}-46$ & $1.67 \mathrm{e}-364$ & 8 & $\mathrm{NS} 4, \theta=0$ & 3 & $5.04 \mathrm{e}-40$ & $1.09 \mathrm{e}-316$ & 8 \\
\hline OSM7 & 3 & $2.25 \mathrm{e}-35$ & $1.13 \mathrm{e}-244$ & 8 & OSM7 & $\mathrm{F}$ & $\mathrm{F}$ & $\mathrm{F}$ & $\mathrm{F}$ \\
\hline OSM8 & $\mathrm{F}$ & F & $\mathrm{F}$ & F & OSM8 & 3 & $6.53 \mathrm{e}-45$ & $4.94 \mathrm{e}-356$ & 8 \\
\hline SAM8 & 3 & $7.68 \mathrm{e}-57$ & $2.94 \mathrm{e}-452$ & 8 & SAM8 & 3 & $8.82 \mathrm{e}-47$ & $1.28 \mathrm{e}-371$ & 8 \\
\hline
\end{tabular}
criteria $\left|x_{n+1}-x_{n}\right| \leq 10^{-15}$ is used to stop the iteration process in all examples.

Table 2. Comparison of various iterative methods. 


\section{Conclusions}

In this paper, new two optimal families of order eight are obtained, based on variants of Ostrowski's method of order seven for solving nonlinear equations. The convergence order of these new families is eight. These new two families (2) and (10) eighth-order have been developed by using weight functions in the third step to increase the order of convergence of method. We have changed the order of convergence of (2) and (10) methods into optimal eight without need to more number of function evaluations. Numerical results are given to illustrate the efficiency and the performance of the new proposed methods.

\section{REFERENCES}

[1] W. Bi, Q. Wu, H. Ren, A new family of eighth-order iterative methods for solving nonlinear equations, Applied Mathematics and Computation, 214 (2009) 236-245. http://dx.doi.org/10.1016/j.amc.2009.03.077

[2] C. Chun, B. Neta, Comparative study of eighth order methods for finding simple roots of nonlinear equations, Numerical Algorithms, 74, (2017), 1169-1201.

[3] C. Chun, B. Neta, On the new family of optimal eighth order methods developed by Lotfi et al., Numerical Algorithms, 72, (2016), 363-376.

[4] C. Chun, YoonMee Ham, Some sixth-order variants of Ostrowski root-finding meth- ods, Applied Mathematics and Computation, $193 \quad$ (2007) 389-394. http://dx.doi.org/10.1016/j.amc.2007.03.074

[5] W. Gautschi, Numerical Analysis: An Introduction, Birkha üser, 1997.
[6] R. King, A family of fourth order methods for nonlinear equations, SIAM, Journal on Numerical Analysis, 10 (1973) 876-879.

[7] J. Kou, Y. Li, Xiuhua Wang, Some variants of Ostrowskis method with seventh-order convergence, Journal of Computational and Applied Mathematics, 209 (2007) 153159. http://dx.doi.org/10.1016/j.cam.2006.10.073.

[8] H. T. Kung and J. F. Traub, "Optimal order of one-point and multipoint iteration," Journal of the Association for Computing Machinery, 21, pp. 643-651, 1974.

[9] L. Liu and X. Wang, Modified Ostrowski's method with eighth-order convergence and high efficiency index, Appl. Math. Letters. 23 (2010) 549-554.

[10] M. S. Petkovic, B. Neta, L. D. Petkovic, J. Dzunic, Multipoint Methods for Solving Nonlinear Equations, Elsevier, 2012.

[11] Sang Deok Lee, Young IkKim, Beny Neta. An optimal family of eighth-order simple-root finders with weight functions dependent on function-to-function ratios and their dynamics underlying extraneous fixed points. Journal of Computational and Applied Mathematics, 317, (2017), $31-54$

[12] J.R. Sharma and H. Arora, A new family of optimal eighth order methods with dynamics for nonlinear equations. Appl. Math. Comput. 273, (2016), 924-933.

[13] J. R. Sharma and R. Sharma, A new family of modified Ostrowski's methods with accelerated eighth-order convergence, Numer. Algor. 54 (2010) 445-458.

[14] Taher Lotfil and Tahereh Eftekhari "A New Optimal Eighth-Order Ostrowski-Type Family of Iterative Methods for Solving Nonlinear Equations" 13 March 2014.

[15] J. F. Traub, "Iterative Methods for Solutions of Equations" Prentice-Hall, Upper Saddle River,1964. 